\title{
Formation, characterization and behaviour of Fe colloids at circumneutral pH at Ainai mine drainage, Japan: Implications from geochemical trends and $\mathrm{Fe}$ isotopes
}

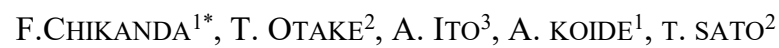

${ }^{1}$ Graduate School of Engineering, Hokkaido University, Sapporo, Japan.

(*correspondence: franceschikanda2@gmail.com)

${ }^{2}$ Faculty of Engineering, Hokkaido University, Sapporo, Japan.

${ }^{3}$ Department of Applied Chemistry for Environment, Kwansei

Gakuin University, Hyogo, Japan.

Colloids, particularly the Fe colloids, have been recognized as significant players in toxic element (im)mobility due to their strong affinity for the metals. Fe colloid formation and behaviour may vary due to the variation of geochemical properties thereby affecting the way they impact the associated metals, and further affecting the sequestration mechanisms of the toxic elements from drainage systems. Various techniques have been applied to understand these behaviors and, in this study, two main fractions of $\mathrm{Fe}$, i.e. dissolved and colloidal fractions have been investigated to clarify the behaviors of $\mathrm{Fe}$ colloids in a circumneutral mine drainage in Japan. Ainai mine drainage is high in Fe and has As and $\mathrm{Zn}$ as other target elements. An abundance of ferrous iron $\left(\mathrm{Fe}^{2+}\right)$ is observed in the upstream, which decreases as we go downstream as aeration occurs thereby resulting in oxidative precipitation of ferric $\mathrm{Fe}\left(\mathrm{Fe}^{3+}\right)$. A clear trend displaying the formation of the colloidal fraction is observed before their aggregation and deposition while the dissolved fraction also decreases. During the formation of the Fe colloids, As displays a similar trend and is removed from the drainage during the deposition of the Fe colloids. Transportation of the homogeneous, Si-bearing ferrihydrite colloids was observed from upstream to downstream before being efficiently removed from the drainage, and their mobility was examined using $\mathrm{Fe}$ isotopes. The total $\mathrm{Fe}$ concentration decreases along with the $\delta^{56} \mathrm{Fe}$ values of the drainage, supporting an isotopic fractionation due to oxidative precipitation. Furthermore, a large fractionation was seen between the two fractions with the colloidal fraction bearing larger $\delta^{56} \mathrm{Fe}$ values due to preferential complexation of heavy $\mathrm{Fe}$ isotopes by hydroxyl ligands to form stronger bonds. The trend displayed by the $\delta^{56} \mathrm{Fe}$ values further suggests significant mobility of colloids in the upstream, although a steep curve towards the downstream also supports significant deposition of the colloids, hence their removal from the drainage. 